\title{
Research on the Product Design of Firefighting Helmet Based on Design Scenario
}

\author{
$\mathrm{Xu}$ Fangting and Hu Kang \\ School of Art \& Design for Wuhan University of Science and Technology \\ Wuhan of Hubei 30065
}

\begin{abstract}
Take firefighting helmet product as object, the situation cognition theory is introduced into product design research. From the perspective of the contextual dynamics of users, products, and environment, this paper further constructs scenario model; analyzes the problems in man-machine systems, and then, in the research of solution scenario, summarizes the design demand of the products in functional domain, physical domain and interaction domain. Then, based on this, this paper also combines the three module characteristics in firefighting helmet products, proposes improved design for the function, structure, form and comfort of products, and provides final design scheme. The design method based on the design scenario theory provides a reference for the design of other related products.
\end{abstract}

Keywords-Product design; Firefighting helmet; Design scenario; Design demand

\section{INTRODUCTION}

Recently, due to the frequent occurrence of fire disasters, this has put forward a higher requirement for the personal security assurance and rescue efficiency of rescue personnel during rescue operation. As the essential protective device for rescue personnel when executing firefighting rescue task, firefighting helmet can exert an important function of protecting the head and the neck. In many fire rescue scenes of our country, the casualty accident of rescue personnel incurred by complicated and varied environment can happen occasionally, and the main reason for such phenomenon is that few attentions are paid to the interactive relationship of people and environment and indistinct problems. On the basis of situation theory, this article has constructed design scenario model, and then through studying the design process of firefighting helmet, it has verified the practicability and effectiveness on the basis of design scenario model.

\section{CONSTRUCt DEsign SCEnARIO MODEL}

With respect to the design scenario, it is a design framework constituted by "behavioral domain-functional domain - physical domain_process domain" mapping relation in axiomatic design [1]. In combination with the research method about people's behaviors and psychologies in psychology and ergonomics, start from design perspective, and then through scientific and reasonable conversion process, convert people's behaviors, psychology, demand, etc. into the final product modeling design characteristics for scenario model construction [2]. The key points emphasized in behavioral domain are the aesthetic demand layer $\{\mathrm{A}\}$ of users for product; the functional domain is reflected in function $\{\mathrm{S}\}$ and restriction conditions $\{C\}$, of which function $\{S\}$ includes meeting the functional demand $\{\mathrm{T}\}$ of physiological safety layer and meeting the demand of users in emotional layer $\{\mathrm{E}\}$; in order to meet the behavioral domain and functional domain in design, create structural parameters $\{D\}$ for product modeling design in physical domain; while the process domain expresses the aforementioned mapping process and problems through several product process variables $\{\mathrm{P}\}$, which are as shown in Figure 1.

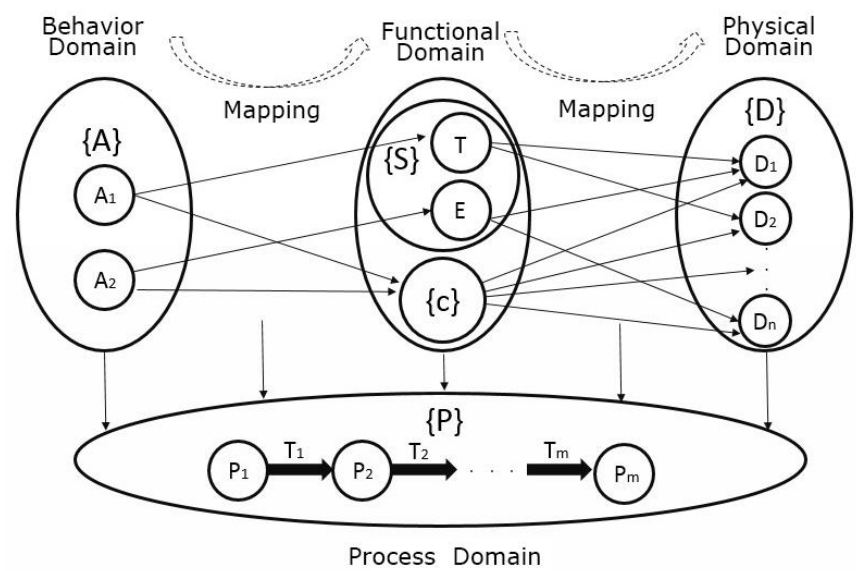

Fig. 1. Design Scenario Framework Diagram 
As can be seen from the design process, the design scenario can be divided into three dimensions, including problem situation, solution situation and result situation, and it includes different statues of the design objective in the design process [3].

$$
\begin{aligned}
\mathrm{sd}= & f(x, y, z), \\
\mathrm{x}= & \{\mathrm{x} 1, \mathrm{x} 2, \ldots, \mathrm{xm}\}, \\
\mathrm{y}= & \{\mathrm{y} 1, \mathrm{y} 2, \ldots, \mathrm{yn}\}, \\
& \mathrm{z}=\{\mathrm{z} 1, \mathrm{z} 2, \ldots, \mathrm{zp}\},
\end{aligned}
$$

In the formula: sd $\neg \longrightarrow$ Design scenario;

$\mathrm{x} \longrightarrow$ Problem scenario;

$\mathrm{y} \longrightarrow$ Solution scenario;

$\mathrm{z} \longrightarrow$ Result scenario.

Changes of $\mathrm{xm}, \mathrm{yn}$ and zp will cause the change of sd, and although factors in $\mathrm{sd}, \mathrm{xm}$, yn and $\mathrm{zp}$ are the same, there are still certain differences.

\section{A. Problem scenario}

In problem scenario, design problems, design restriction and restriction conditions are the key points for scenario research. Through studying the interaction mode of various scenario factors for the user and product during the period, when it comes to different scenarios, the behavior cognition and emotions of users are different, which will directly influence the final value of product. As per the scenario factors, with respect to the characteristics of product modeling, the design problem scenario is mainly from design requirements, result scenario is the final design scheme, and design behavior can be mostly reflected as a kind of design strategy. Thus, the design case of one product can be simply expressed as follows:

$$
\mathrm{Cimi}=(\mathrm{Cs}, \mathrm{P}, \mathrm{K}, \mathrm{U})
$$

In the formula: Cs_—Relationship;

$\mathrm{P} \_$Problem situation;

$\mathrm{K}-$ Strategy;

U—Result scenario.

\section{B. Solution scenario}

The solution scenario refers to a process for the designer to convert design problems into design result as per the design information and design problems in combination with the design experience. Solution scenario refers to the description about the design process behavior, and it is a process for designing external knowledge into product. In the solution scenario, the design strategy and solution steps as well as design methods, etc. for the designer are the key points for scenario expression. The solution scenario can be expressed as:

$$
\mathrm{Cv}=(\mathrm{S}, \mathrm{P})
$$

In the formula: $\mathrm{Cv} \longrightarrow$ Solution scenario;

$$
\begin{aligned}
& \mathrm{S} \longrightarrow \text { Solution mode; } \\
& \mathrm{P} \longrightarrow \text { Problem scenario. }
\end{aligned}
$$

Through analyzing the problem scenario of firefighting helmet product, study from three aspects in product solution scenario:

(1) Problems based on users; since demand has fuzziness, the cognitive capacity of rescue personnel is limited, and since the fire disaster rescue activity behavior is extremely dangerous, it is quite important to ensure the life safety of rescue personnel; besides, goods user experience is also the key points emphasized during the design.

(2) Problems based on products; there is only the reasonable matching of parts and the whole, and it includes the physical domain form of product, man-machine size, color and other series of factors, so as to better exert product functions.

(3) Problems based on environment; due to complicated and varied fire disaster environment, in the fire scene covered with dense smoke, the sight of rescue personnel will be dim, and this will cause big barrier for rescue task; besides, flames, gravels, and heavy objects will also cause emergency dangers to the rescue personnel [4].

\section{Result scenario}

Result scenario is the description about the objective status of design problems, and is the final result of design knowledge feedback. Through putting forward problem scenario, analyzing solution scenario, and obtaining the design demand for functional domain, physical domain and interaction domain, so as to provide design scheme. The result scenario can be simply expressed as follows:

$$
\mathrm{U}=(\mathrm{Ff}, \mathrm{Fp}, \mathrm{Fj})
$$

In the formula: $\mathrm{U} \longrightarrow$ Result scenario;

$$
\begin{aligned}
& \mathrm{Ff}-\text { Functional domain; } \\
& \mathrm{Fp} \text { __ Physical domain; } \\
& \mathrm{Fj} \text { _- Interaction domain }
\end{aligned}
$$

Through studying the solution scenario of firefighting helmet product, obtain the key points that shall be noticed for the result scenario design of product:

(1) Functional domain; specific to the insufficient functions of current firefighting helmet, put forward function improvement scheme and increase new model function in combination with new technologies.

(2) Physical domain; confirm the product form, structure, size, color and other factors as per the function and using environment characteristics of firefighting helmet.

(3) Interaction domain; optimize the control and wearing comfort of users considering the operation procedures of firefighting helmet, and reinforce the information transmission and receiving between users and environment. 


\section{STRUCTURAL SYSTEM}

As a product that can adapt to specific environment and group, the entity function relationships for the control factors of firefighting helmet are extremely clear, and on this basis, corresponding module division is conducted [5].

\section{A. Safety protection module}

Head is the most important part of human body, and also the most vulnerable part that can mostly easily get hurt, so in order to realize the best protection, when designing the helmet, it is requested to follow the national standard requirements of GA44-2015 Firefighting Helmet [6], which are as shown in Table 1.

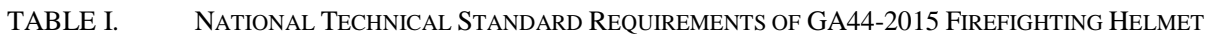

\begin{tabular}{cr}
\hline Factor & National Technical Standard Requirements \\
Impact force & Maximum value $\leq 3780 \mathrm{~N}$ \\
Fire resistance & The shell flame burning time is within $5 \mathrm{~s}$ \\
Metal parts & $\begin{array}{c}\text { Smooth surface, without point corrosion pit } \\
\text { Mask light transmittance }\end{array}$ \\
Helmet mass (excluding the cape and accessories) & $\begin{array}{c}\text { mask } \geq 85 \% \\
\text { Mass } \leq 1800 \mathrm{~g}\end{array}$
\end{tabular}

The form of helmet shell should match with the head form of rescue personnel, please refer to the modeling regulations about helmet shell protection range in GA44-2015 Firefighting
Helmet[6] for design, of which the protection range for "fullhelmet type" helmet is the part above ACDEF line, which are as shown in Figure 2.

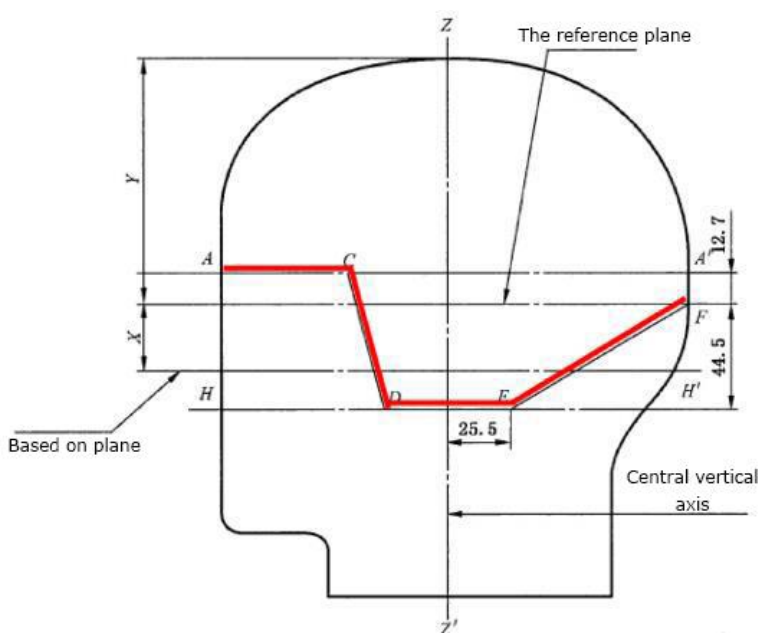

Fig. 2. Safety Protection Range

\section{B. Illumination module}

When accidents occur, due to the special character of fire disaster, this will often generate lots of danger, especially at night, the illumination module can not only exert the task of rescue illumination, but also the task of directional light rescue. The use of integrated structure illumination device can integrate the machine core, eyepiece, battery, etc. into an organic whole, and then use connection fixation device for assembly. Integrated structural illumination device: simple structure, without complicated connection mechanism, easy to operate, with high reliability; conduct overall protection, better realize overall high temperature resistance, waterproof, antidropping and other performances; currently, domestic and overseas military, firefighting and other industrial helmet illumination mostly adopt such structure; to some extent, integrated structural illumination can liberate the two-hand burden of rescue personnel. Thus, the firefighting helmet product can adopt integrated structure illumination mode for design, which is as shown in Figure 3.

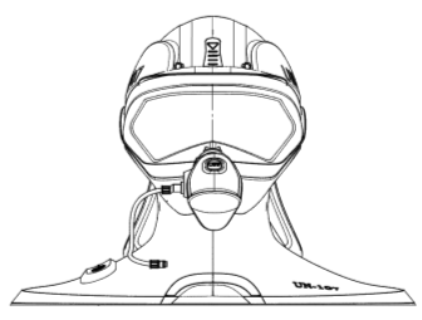

Fig. 3. Integrated Structure Illumination Devices 


\section{Information transmission module}

Except these, the helmet can also possess wireless communication technology, through the helmet, it is applicable to conduct voice transmission and image transmission for the fire accident site, and complete data application analysis. Except these, it is also requested to position and monitor the body of the rescue personnel, understand the moving status and body energy conditions of rescue personnel, realize the alarm of poisonous materials, timely master the life symbols of rescue personnel, and then on the basis of ensuring people's life safety, it is also requested to pay attention to the life safety of rescue personnel.

\section{SCHEME DESIGN}

As per the rescue conditions, conduct design scenario analysis for various functional modules of firefighting helmet, so as to obtain the improvement design scheme of all parts. And then combine all of these, further perfect the overall modeling design of firefighting helmet, to obtain the optimal scheme [7].

\section{A. Functional design}

While ensuring the head safety of rescue personnel, firefighting helmet can also solve problems related to illumination and information transmission during rescue operation. During the design, it has been equipped with assembly and disassembly neck protection cape, which can better conduct safety protection for the shoulder and the neck. The cape assembly is at the lower edge of firefighting helmet, which can protect the neck and ears of rescue personnel from being infringed by external fluid or thermal radiation; besides, the cape is made of fabrics with fire resistance, heat resistance and waterproof performance, and the mass is relatively light; meanwhile, it will not influence the flexibility of body parts, obtain the objective of comfortable wearing, which is as shown in Figure 4.

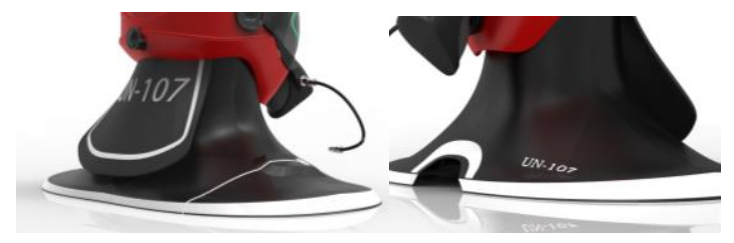

Fig. 4. Firefighting Helmet Cape Modeling

When being compared with the former traditional firefighting equipment, rescue personnel will encounter with many restriction conditions during emergency rescue activity, so they cannot complete the task with high efficiency. This time, the design of firefighting helmet is internally equipped with AR glasses, and while exerting safety protection functions, it can also project the fire scene on the mirror plane, and facilitate the rescue personnel to take actions as per conditions; the helmet shell is set with respirator device interface; it adopts the mode of plug and play, and can provide many conveniences for rescue, which is as shown in Figure 5.

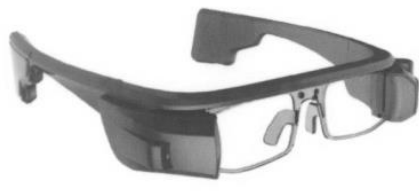

(a)AR glass

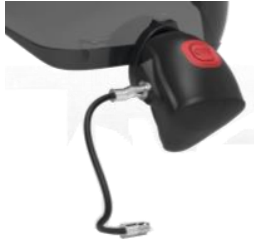

(b) Respirator device
Fig. 5. Firefighting Helmet Functional Parts Drawing

\section{B. Structure and form design}

As per the product functions, confirm the product form and structure [8]. As can be seen in Figure 6, the firefighting helmet is mainly constituted by helmet shell, breathing mask, protective mask, eyes protector, illumination module, and protective cape. The helmet adopts modularized design concept, and can facilitate the timely replacement of task module under different environment scenarios, and complete operation efficiently. Due to high modularization, the entire firefighting helmet system interface has universality, and can create conditions for the repair and replacement upgrading of followup equipment system.

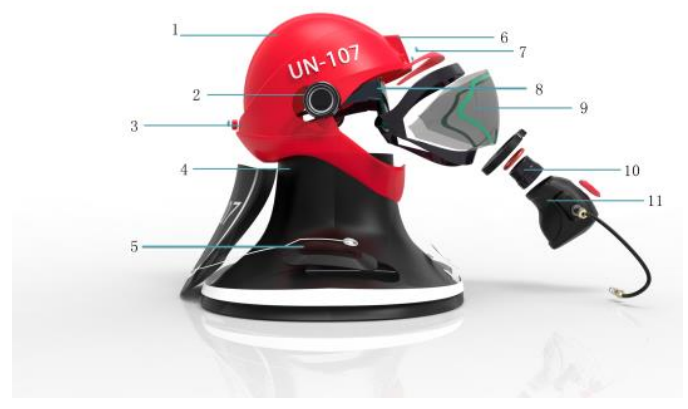

Fig. 6. Structural Design Drawing

1. Helmet shell; 2. Bone conduction headset; 3. Postposition camera; 4. Protective cape;5. Circuit board; 6 . Infrared LED lamp; 7. Camera; 8 AR glasses; 9. Protective mask; 10. Respirator connector; 11. Respirator device

The helmet shell materials can meet the requirements of high temperature resistance, flame resistance, shock resistance, penetration resistance and other performances. While colors are endowed with specific emotions and connotations in product design, and can arouse emotions and express feelings, and even influence people's physiological feelings[9]. Since rescue devices should make people feel safe with high differentiation degree, red is mostly adopted because of its high lightness and purity during design, and can exert a striking effect, which is as shown in Figure 7. 


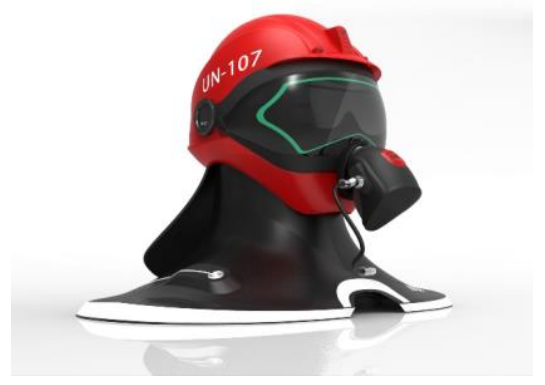

Fig. 7. Product Effect Drawing

\section{Comfort design}

The comfort of firefighting helmet is related to many factors, the firefighting helmet is internally set with transparent goggle parts with anti-shock performance, and while reinforcing the thermal radiation protection performance, this can decrease the mass of firefighting helmet to a certain degree, and increase the comfort; the heavier the mass of helmet is, the worse the comfort will be; while the main part of mass is the helmet shell, the improvement of helmet shell materials, structures and technologies can decrease the mass, and improve comfort. Under certain mass, the more even the head pressure distribution is, the better the comfort will be. Thus, during the design, it is requested to pay attention to the contacted area for the helmet and the head, the part, and flat fitting degree, and then design as per the average parameters of the group, and reinforce the goodness fit for the helmet model and the human head form and size, which are as shown in Figure 8.
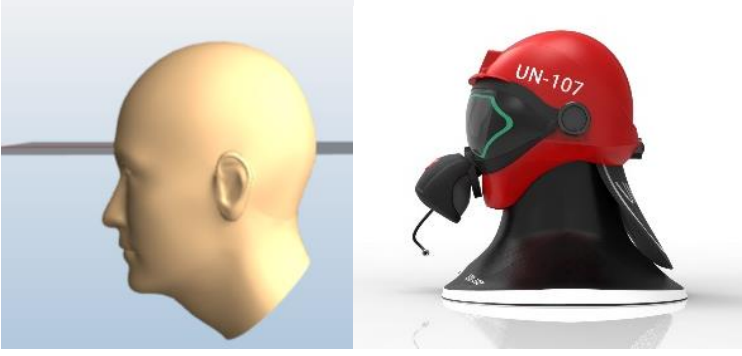

Fig. 8. External Modeling Drawing of Firefighting Helmet

\section{CONCLUSION}

This article is based on the method of design scenario, and takes firefighting helmet product as the design object, constructs the design scenario model, and then through the research about the design process of firefighting helmet product, it has verified the effectiveness and application value based on design scenario model, and the specific conclusion is as follows:

(1) The research about the product design of firefighting helmet based on design scenario can provide a scenario dynamic analysis for the product design of firefighting helmet through taking "user-product - environment" as the cutting point. Through analyzing problem scenario, it has integrated solution scenario and then confirmed the final design scheme, and conduct the design of various functional parts of helmet in combination with new technologies, make the function more reasonable and better meet the physiological and psychological demands of users.

(2) Start from functional demand, structural and form demand, comfort demand, solve the problems existed in current products, meet the new demand of rescue personnel, and also provide new design thought and methods for the design of other relevant firefighting protection products.

\section{REFERENCES}

[1] Tan Hao, Zhao Jianghong, Wang Wei et al. Industrial Design Scenario Model Based on Cases and the Application [J]. Journal of Mechanical Engineering, 2006 (12): 151-157.

[2] [US] Carl T. Ulrich, Stephen D. Eppinger. Product Design and Development [M]. Translated by Yang Qing et al. Beijing: China Machine Press, 2015.

[3] Luo Shijian, Zhu Shangshang, Ying Fangtian et al. User Experience Design Based on Scenario in Mobile Phone Interface [J]. Computer Integrated Manufacturing System, 2010(2): 239-243

[4] Xu Shihu, Zhou Zhen and Xia Jinjun. Firefighting Rescue Individual Equipment Concept Design [J]. Mechanical Design, 2016 (2): 122-125.

[5] Ma Jiaojiao. Development of New Firefighting Helmet [D]. Shanghai: Shanghai Jiaotong University, 2007.

[6] GA 44-2015 Public Safety Industry Standards of PRC Firefighting Helmet [S].

[7] He Yuan, Cheng Xufeng, Zhang Shanwei et al. Research about the Design of Household Smoke Alarm [J]. Mechanical Design, 2016 (9): 105-108.

[8] Ye Donghai and Zhou Zhen. Research about the Design of Upper Body Protection Armor for Firemen Based on Material Characteristics [J]. Mechanical Design, 2014 (1): 113-116.

[9] He Xiaomei, Zhang Lulu and Nie Lu. Research about the Design of Flood Emergency Rescue Package Based on UCD [J]. Packaging Engineering, 2011 (6): 26-29. 